Case Report

\title{
Metastatic Melanoma to the Urinary Bladder of Ocular Origin Accompanied with Primary Cutaneous Melanoma: Diagnostic Challenge-A Report of a Case
}

\author{
Constantine Theocharides, ${ }^{1}$ Kyriakos Chatzopoulos, ${ }^{1}$ Dimitrios Papanikolaou, ${ }^{2}$ \\ Vasileios Siokas, ${ }^{1}$ Ioannis Amplianitis, ${ }^{3}$ and Athanasios Papanikolaou ${ }^{1}$ \\ ${ }^{1}$ Department of Pathology, “Gennimatas” General Hospital, Thessaloniki, Greece \\ ${ }^{2}$ Department of Urology, Papageorgiou General Hospital, Thessaloniki, Greece \\ ${ }^{3}$ Department of Pathology, "Hippokration" General Hospital, Thessaloniki, Greece \\ Correspondence should be addressed to Athanasios Papanikolaou; apap250@gmail.com
}

Received 19 January 2017; Revised 26 May 2017; Accepted 2 August 2017; Published 11 September 2017

Academic Editor: Ondrej Hes

Copyright ( 2017 Constantine Theocharides et al. This is an open access article distributed under the Creative Commons Attribution License, which permits unrestricted use, distribution, and reproduction in any medium, provided the original work is properly cited.

\begin{abstract}
Metastases of melanoma to the urinary bladder are infrequent. Even rarer are metastases to the urinary bladder from uveal melanoma, with only 3 cases published in the literature so far. Herein we present a case of a 77-year-old male patient who presented with metastatic melanoma to the urinary bladder. The patient's history included the diagnoses of uveal melanoma treated with radiation 25 years ago, as well as that of cutaneous melanoma diagnosed 7 years ago. The molecular study of the urinary bladder tumor specimen identified mutation of the GNAQ gene, which has been suggested to be an early molecular event in the pathogenetic course of over $80 \%$ of uveal melanomas. Therefore, the diagnosis of uveal melanoma metastatic to the urinary bladder was made.
\end{abstract}

\section{Introduction}

The metastatic tumors to urinary bladder are extremely rare [1-5]. Given the infrequency of the metastatic tumors to the urinary bladder and due to the fact that malignant melanomas consist of a subgroup of the specified tumors, metastatic melanoma to urinary bladder is designated an even more rare histopathological entity. The fact that primary malignant melanoma of urinary bladder is also a very rare lesion [6-8] must also be taken into consideration in the differential diagnosis of a melanocytic lesion of the urinary bladder [9-11].

Metastatic melanomas to urinary bladder used to be mainly autopsy findings [1,11] and currently, apart from case reports and small case series, no comprehensive cohorts of patients with metastatic melanoma to the urinary bladder have been reported [3,12]. Both the great rarity and the limited literature references make metastatic melanomas to urinary bladder a serious diagnostic challenge for histopathologists and raise severe diagnostic issues on the differential diagnosis from other urinary bladder neoplasms. The determination of the original focus of metastatic melanomas to urinary bladder is convenient in some cases, where the primary focus is already known from patient's reported clinical history. However, when two independent primary foci of malignant melanoma coexist or have already been manifested, the determination of origin is a challenge by itself and exceedingly difficult to correlate the separate histological findings. The contribution of contemporary methods of molecular pathology is considered more than necessary.

In the presenting case, the persisting diagnostic challenge is discussed as a potential association of a recent melanocytic lesion in the urinary bladder with either a cutaneous melanoma which occurred seven years ago or an ocular melanoma which occurred twenty-five years ago. The possibility of correlation between all these three involved anatomic sites cannot be easily rejected. 
TABLE 1: Panel of antibodies used in the present case report. RTU: ready to use.

\begin{tabular}{lccc}
\hline Antibody & Clone & Company & Dilution \\
\hline Anti-Melan A & A103 & BioGenex, USA & RTU \\
Anti-S100 & AR058-10R & BioGenex, USA & RTU \\
Anti-Melanosome & HMB45 & Zeta Corporation, USA & $1: 100$ \\
Anti-Vimentin & V9 & BioGenex, USA & RTU \\
Anti-Desmin & DE-R-11 & Leica Biosystems, UK & RTU \\
Anti-Chromogranin A & $5 \mathrm{H} 7$ & Leica Biosystems, UK & RTU \\
Anti-EMA & GP1.4 & Thermo Fisher Scientific, UK & RTU \\
Anti-CD99 & $12 E 7$ & Leica Biosystems, UK & RTU \\
Anti-Keratin & AE1/AE3 & Zeta Corporation, USA & $1: 100$ \\
\hline
\end{tabular}

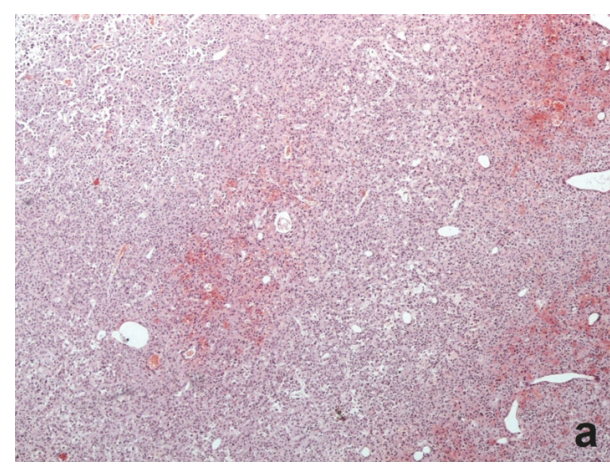

(a)

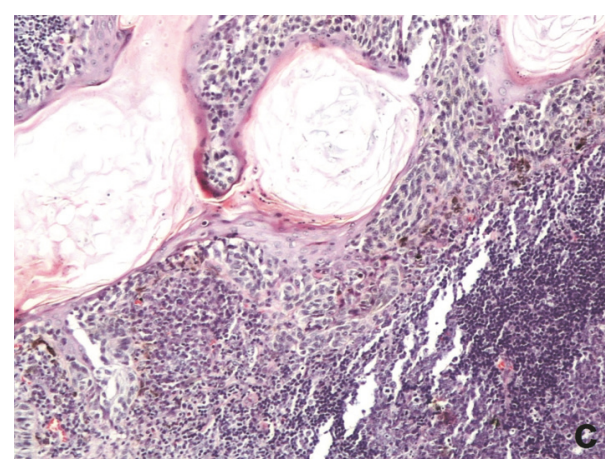

(c)

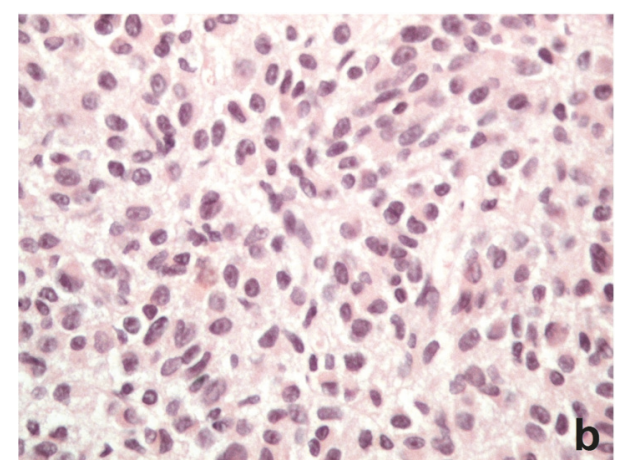

(b)

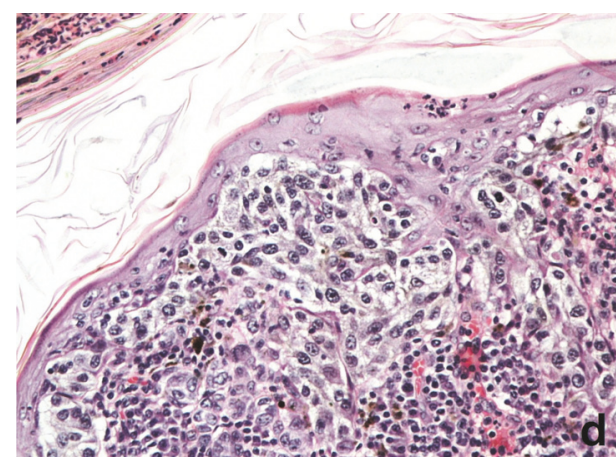

(d)

FIgURE 1: Histopathologic features of the urinary bladder tumor $(a, b)$ and the cutaneous lesion (c, d).

\section{Case Presentation}

A 77-year-old male had been diagnosed with ocular melanoma 25 years ago and had been subjected to radiotherapy. Seven years ago, a cutaneous lesion appeared and it had been identified as malignant cutaneous melanoma. In both occasions, diagnostic and therapeutic procedures were performed at different hospitals. The associated histopathology report which referred to ocular melanoma was not available in patient's medical record. However, the previous histopathology report, relative to cutaneous melanoma, was provided.

The patient underwent transurethral resection (TURBT) of a polypoid lesion in urinary bladder which resected all the apparent gross lesion with gross total resection of $3 \mathrm{~cm}^{3}$ tumor volume. During microscopic examination of the
TURBT specimen, the presence of malignant tumor segments was ascertained, which were characterized by high cellular density areas. The cytological features of tumor cells included large nuclei with variation in size and prominent nucleoli (Figures 1(a) and 1(b)). Immunohistochemical expressions (Table 1) of S100 protein, Melan A (Figure 2), HMB45, and Vimentin were positive, while expressions of Cytokeratin AE1/AE3, Desmin, Chromogranin, and CD99 were negative. It is worth mentioning that in the examined specimen there were detected areas of normal mucosa (transitional epithelium) with neither dysplastic lesions nor in situ carcinoma.

According to the available previous histopathology report, in relation to the cutaneous melanoma which occurred seven years ago, the diagnosis was the one of superficial spreading melanoma. The surgical specimen received at 
TABLE 2: Clinical features of the 4 cases of uveal melanoma metastases in the urinary bladder that have been reported so far in the literature.

\begin{tabular}{lccccc}
\hline Publication & Age & Gender & Concurrent metastases & $\begin{array}{c}\text { Years after initial uveal } \\
\text { melanoma diagnosis }\end{array}$ & Clinical course \\
\hline Moore et al. [27] & 82 & Male & None & 12 & Free of disease at 9 months \\
Irisawa et al. [28] & 77 & Male & Widespread & 6 & Death at 10 months \\
Wisenbaugh et al. [29] & 62 & Female & Widespread & 10 & Death at 5 months \\
Present case & 77 & Male & Widespread & 25 & Widespread multiple metastases at months \\
\hline
\end{tabular}

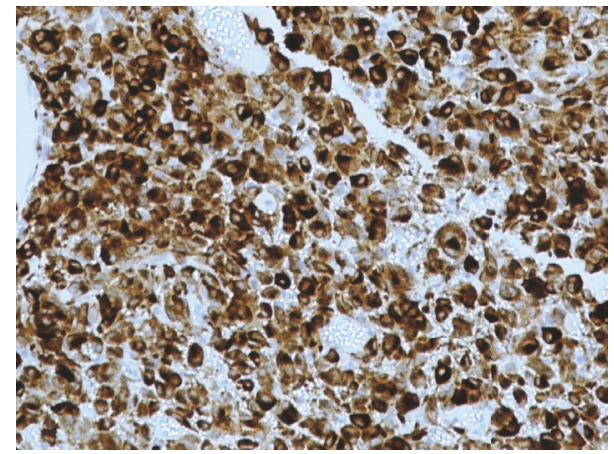

Figure 2: Melan A immunopositivity in the cells of the TURBT specimen.

that time was a skin ellipse measuring $2.6 \times 1.4 \times 0.4 \mathrm{~cm}$ with raised, pigmented lesion measuring $1,7 \mathrm{~cm}$ in greater dimension. More specifically, there was a malignant melanocytic lesion mainly in horizontal growth phase with focal vertical growth, which infiltrated the papillary dermis, while Breslow thickness was estimated at $0.45 \mathrm{~mm}$ and evaluated as Clark's level III (Figures 1(c) and 1(d)). Immunohistochemical markers were positive for S100, Melan A, and HMB45 and negative for Cytokeratins and EMA. The lesion was excised within healthy tissues.

Afterwards and subsequent to the initial specimen examination, consultation was requested and a paraffin block of the TURBT specimen was sent for secondary opinion and further examination due to the probable correlation between the aforementioned melanocytic tumors and the recent bladder tumor. According to the consultation report, there was concordance over the morphological and the immunohistochemical findings of the initial examination. The existence of melanoma in vertical phase growth was also confirmed with necrotic areas and considerable ulcerative surface while Breslow thickness was estimated at least $0.55 \mathrm{~mm}$. The neoplastic cell population was dominated by epithelioid type cells with focally severe atypia which conveyed plasmacytoid features. In some areas, single giant, multinucleated, or binucleated cellular forms were also seen with sporadic intranuclear inclusions. The MIB1/Ki-67 index exhibited a wide range from $3 \%$ to $15 \%$. At the same time, genetic analysis was performed for the BRAF, GNA11, GNAQ, and C-KIT mutation status. The genetic analysis proved positive for the exon 4 c.548G $>$ A p.R183Q GNAQ mutation while it provided negative results for the rest of all.

\section{Discussion}

The diagnostic challenge lied in the probable correlation between the three melanocytic lesions which occurred in three different anatomic sites. Despite the fact that urinary bladder constitutes an extremely uncommon metastasis site for both ocular and cutaneous malignant melanomas [1316], the patient had remained free of metastatic disease for an interval of 25 years. This timeframe is considered to be too long for the emergence of distant metastasis [17, 18]. The epithelioid cells detected in the TURBT specimen exhibited some morphological features similar to those of epithelioid type of uveal malignant melanoma, but given that the specific histological type predicts more aggressive behavior [19], the metastasis free interval of 25 years could not be easily justified. Nevertheless, it has been reported that ocular melanoma may exhibit metastasis free intervals longer than 25 years $[20,21]$.

Intraocular melanoma may occur at any point of the uveal tract. Patient survival is directly associated with the histological type of the primary ocular focus, which is designated as the most important parameter, while the survival rate seems to be higher for spindle cell type in contrary to epithelioid type [19]. It is also worth mentioning that some researchers classify spindle cell melanoma as uveal nevus [22]. According to existing literature, additional prognostic features are tumor size at primary focus, intraocular localization, optic nerve involvement, tumor necrosis presence or absence, tumor neovascularization, and lymphocytic infiltration, as well as nuclear features of neoplastic cell population [23-25]. By order of priority, uveal melanoma gives metastases to liver, lungs, bones, and skin [14, 23]. According to the literature data, manifestation of distant metastases varies and their occurrence may emerge even after many years since eye enucleation and supplementary radiotherapy or/and chemotherapy [20, 21]. Furthermore, existing evidence regarding metastasis from ocular melanoma with morphological features simulating blue nevi [26] made the diagnostic challenge even more complicated. In order to get more insight about the physical course of metastasizing uveal melanomas to the urinary bladder, we performed a literature search for previously published cases. Only four cases have been reported so far, including the present case. All of them included patients of older age, who presented with urinary bladder metastases from 6 to 25 years after the initial diagnosis of uveal melanoma (Table 2).

The molecular examination proved to be of paramount importance for making the correct diagnosis. According to the best of our knowledge, this is the first case report which 
used molecular examination to distinguish whether the primary focus of a metastatic lesion of the urinary bladder was ocular or cutaneous. In fact, it has been suggested that GNAQ mutations are an early event characterizing over $80 \%$ of uveal melanomas [30, 31]. Therefore, the presence of that specific mutation in the material retrieved from the TURBT specimen confirmed the fact that the metastasis to the urinary bladder originated from the uveal melanoma diagnosed 25 years ago. At the time of writing (five months after TURBT), the patient has been diagnosed with multiple metastases to liver and spleen, as well as to celiac and mediastinal lymph nodes.

\section{Conflicts of Interest}

The authors declare no conflicts of interest regarding the publication of this paper.

\section{References}

[1] J. E. Meyer, "Metastatic melanoma of the urinary bladder," Cancer, vol. 34, no. 5, pp. 1822-1824, 1974.

[2] A. Paterson, M. Sut, A. Kaul et al., "Metastatic malignant melanoma of the urinary bladder: case report and literature review," Central European Journal of Urology, vol. 65, no. 4, pp. 232-234, 2012.

[3] V. Velcheti and R. Govindan, "Metastatic cancer involving bladder: a review," The Canadian Journal of Urology, vol. 14, no. 1, pp. 3443-3448, 2007.

[4] A. Rishi, T. A. Anderson, A. M. Kirschenbaum, and P. D. Unger, "Metastatic malignant melanoma to urinary bladder: a potential pitfall for high-grade urothelial carcinoma," International Journal of Surgical Pathology, vol. 22, no. 4, pp. 347-351, 2014.

[5] G.-Q. Xiao, J. Chow, and P. D. Unger, "Metastatic tumors to the urinary bladder: Clinicopathologic study of 11 cases," International Journal of Surgical Pathology, vol. 20, no. 4, pp. 342-348, 2012.

[6] W. E. Khalbuss, M. Hossain, and A. Elhosseiny, "Primary malignant melanoma of the urinary bladder diagnosed by urine cytology: A case report," Acta Cytologica, vol. 45, no. 4, pp. 631635, 2001.

[7] P. Dahm and J. E. Gschwend, "Malignant non-urothelial neoplasms of the urinary bladder: a review," European Urology, vol. 44, no. 6, pp. 672-681, 2003.

[8] M. Pacella, F. Gallo, C. Gastaldi, C. Ambruosi, and G. Carmignani, "Primary malignant melanoma of the bladder," International Journal of Urology, vol. 13, no. 5, pp. 635-637, 2006.

[9] A. M. Ainsworth, W. H. Clark, M. Mastrangelo, and K. B. Conger, "Primary malignant melanoma of the urinary bladder," Cancer, vol. 37, no. 4, pp. 1928-1936, 1976.

[10] H. M. Tainio, T. M. Kylmälä, and H. K. Haapasalo, "Primary malignant melanoma of the urinary bladder associated with widespread metastases," Scandinavian Journal of Urology and Nephrology, vol. 33, no. 6, pp. 406-407, 1999.

[11] H. Van Ahlen, V. Nicolas, W. Lenz, A. Bockisch, I. Boldth, and W. Vahlensieck, "Primary melanoma of urinary bladder," Urology, vol. 40, no. 6, pp. 550-554, 1992.

[12] A. W. Bates and S. I. Baithun, "Secondary neoplasms of the bladder are histological mimics of nontransitional cell primary tumours: clinicopathological and histological features of 282 cases," Histopathology, vol. 36, no. 1, pp. 32-40, 2000.
[13] M. N. Sirott, D. F. Bajorin, G. Y. C. Wong et al., "Prognostic factors in patients with metastatic malignant melanoma: A multivariate analysis," Cancer, vol. 72, no. 10, pp. 3091-3098, 1993.

[14] S. Rajpal, R. Moore, and C. P. Karakousis, "Survival in metastatic ocular melanoma," Cancer, vol. 52, no. 2, pp. 334-336, 1983.

[15] E. S. Gragoudas, K. M. Egan, J. M. Seddon et al., "Survival of patients with metastases from uveal melanoma," Ophthalmology, vol. 98, no. 3, pp. 383-390, 1991.

[16] C. M. Balch, "Cutaneous melanoma: prognosis and treatment results worldwide," Seminars in Surgical Oncology, vol. 8, no. 6, pp. 400-414, 1992.

[17] Collaborative Ocular Melanoma Study Group, "Development of metastatic disease after enrollment in the COMS trials for treatment of choroidal melanoma," Archives of Ophthalmology, vol. 123, no. 12, pp. 1639-1643, 2005.

[18] C. L. Shields, "Conjunctival melanoma: Risk factors for recurrence, exenteration, metastasis, and death in 150 consecutive patients," Transactions of the American Ophthalmological Society, vol. 98, pp. 471-492, 2000.

[19] T. Sakamoto, M. Sakamoto, H. Yoshikawa et al., "Histologic findings and prognosis of uveal malignant melanoma in Japanese patients," American Journal of Ophthalmology, vol. 121, no. 3, pp. 276-283, 1996.

[20] J. C. Allen and W. H. Jaeschke, "Recurrence of Malignant Melanoma in an Orbit After 28 Years," Archives of Ophthalmology, vol. 76, no. 1, pp. 79-81, 1966.

[21] J. A. Shields, J. J. Augsburger, L. A. Donoso, V. B. Bernardino Jr., and M. Portenar, "Hepatic metastasis and orbital recurrence of uveal melanoma after 42 years," American Journal of Ophthalmology, vol. 100, no. 5, pp. 666-668, 1985.

[22] J. P. Ganley and G. W. Comstock, "Benign nevi and malignant melanomas of the choroid," American Journal of Ophthalmology, vol. 76, no. 1, pp. 19-25, 1973.

[23] I. W. McLean, V. S. Saraiva, and M. N. Burnier, "Pathological and prognostic features of uveal melanomas," Canadian Journal of Ophthalmology / Journal Canadien d'Ophtalmologie, vol. 39, no. 4, pp. 343-350, 2004.

[24] R. Folberg, V. Rummelt, R. P.-V. Ginderdeuren et al., “The prognostic value of tumor blood vessel morphology in primary uveal melanoma," Ophthalmology, vol. 100, no. 9, pp. 1389-1398, 1993.

[25] P. O. L. De Cruz, C. S. Specht, and I. W. McLean, "Lymphocytic infiltration in uveal malignant melanoma," Cancer, vol. 65, no. 1, pp. 112-115, 1990.

[26] J. S. Wieselthier and W. L. White, "Cutaneous metastasis of ocular malignant melanoma: An unusual presentation simulating blue nevi," American Journal of Dermatopathology, vol. 18, no. 3, pp. 289-295, 1996.

[27] N. A. Moore, H. J. Stunell, B. Sizer, and R. G. Casey, "Malignant melanoma metastasis to the urinary bladder: a rare cystoscopic finding," Journal of Clinical Urology, vol. 8, no. 2, pp. 143-146, 2015.

[28] C. Irisawa, Y. Onmura, and S. Matsushita, "Metastatic malignant melanoma of the urinary bladder: a case report," Hinyokika Kiyo. Acta Urologica Japonica, vol. 33, no. 3, pp. 424-427, 1987.

[29] E. S. Wisenbaugh, M. L. Stanton, G. M. Grimsby, M. D. Tyson, and E. P. Castle, "Metastatic malignant melanoma to the bladder: A case series," Current Urology, vol. 6, no. 1, pp. 53-56, 2012. 
[30] M. D. Onken, L. A. Worley, M. D. Long et al., "Oncogenic mutations in GNAQ occur early in uveal melanoma," Investigative Ophthalmology and Visual Science, vol. 49, no. 12, pp. 52305234, 2008.

[31] C. D. van Raamsdonk, V. Bezrookove, and G. Green, "Frequent somatic mutations of GNAQ in uveal melanoma and blue naevi," Nature, vol. 457, no. 7229, pp. 599-602, 2009. 


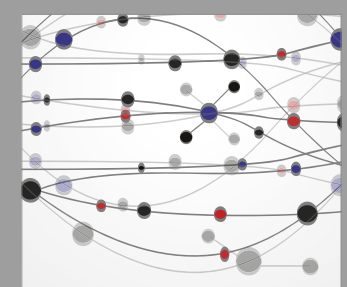

The Scientific World Journal
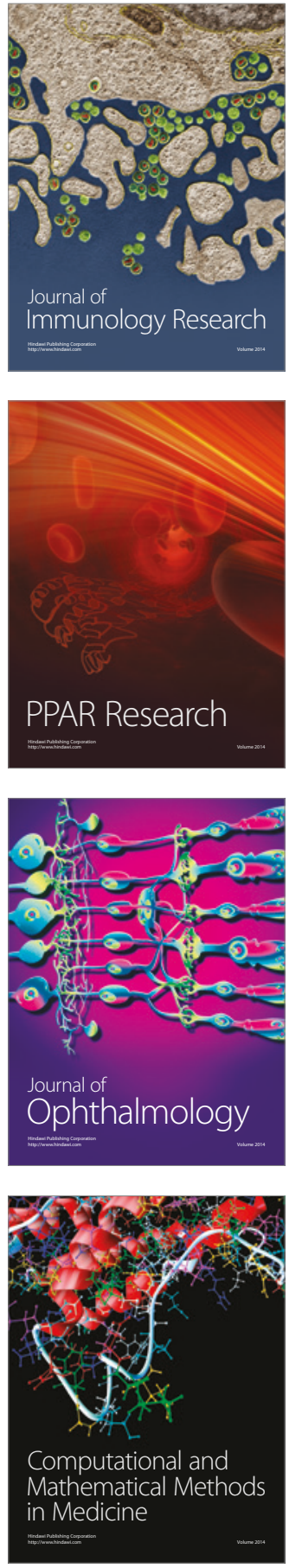

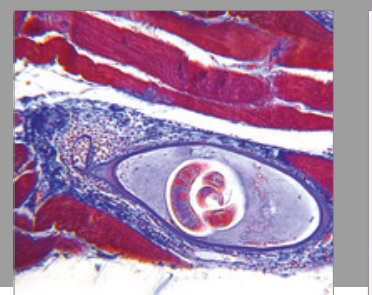

Gastroenterology Research and Practice
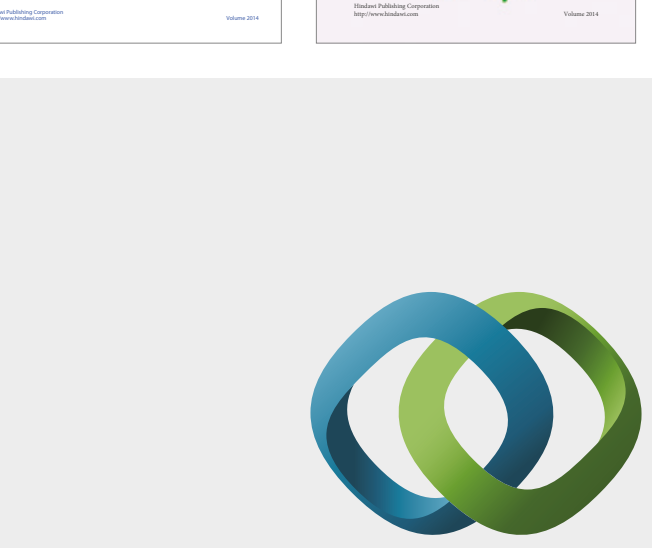

\section{Hindawi}

Submit your manuscripts at

https://www.hindawi.com
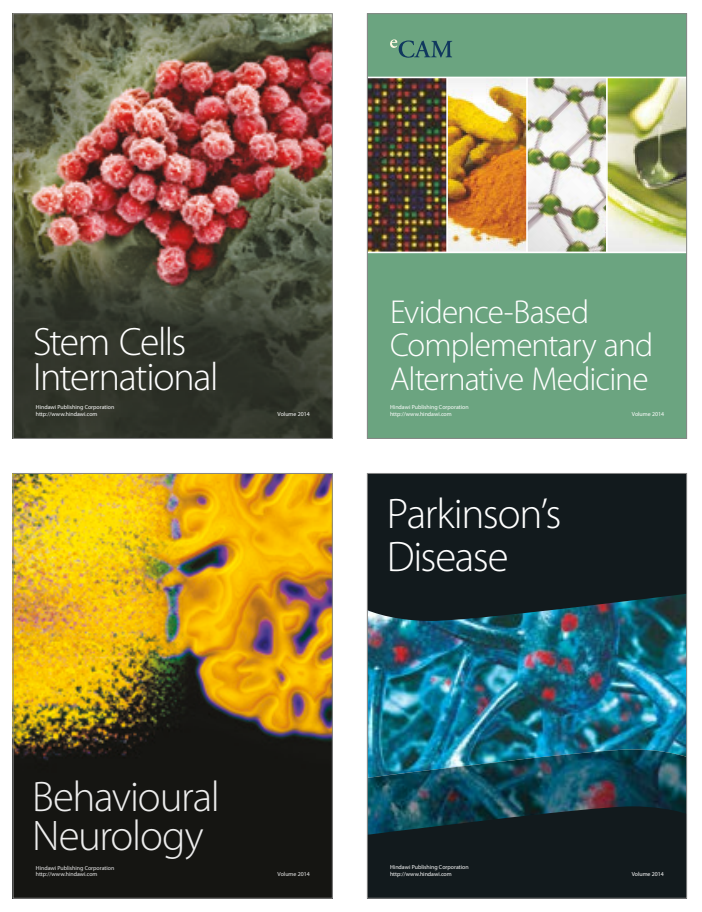
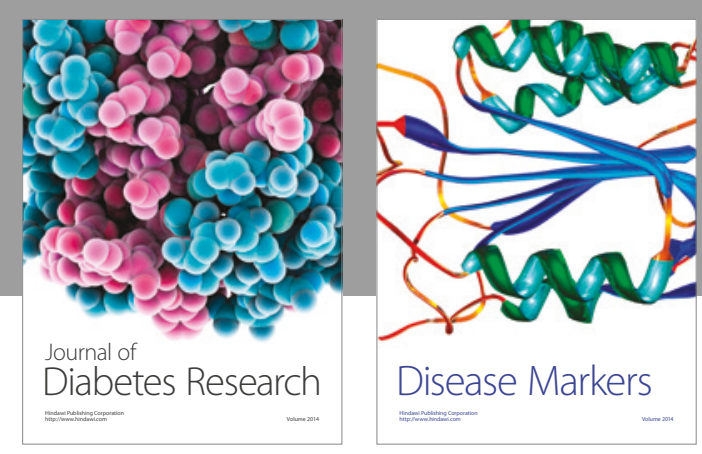

Disease Markers
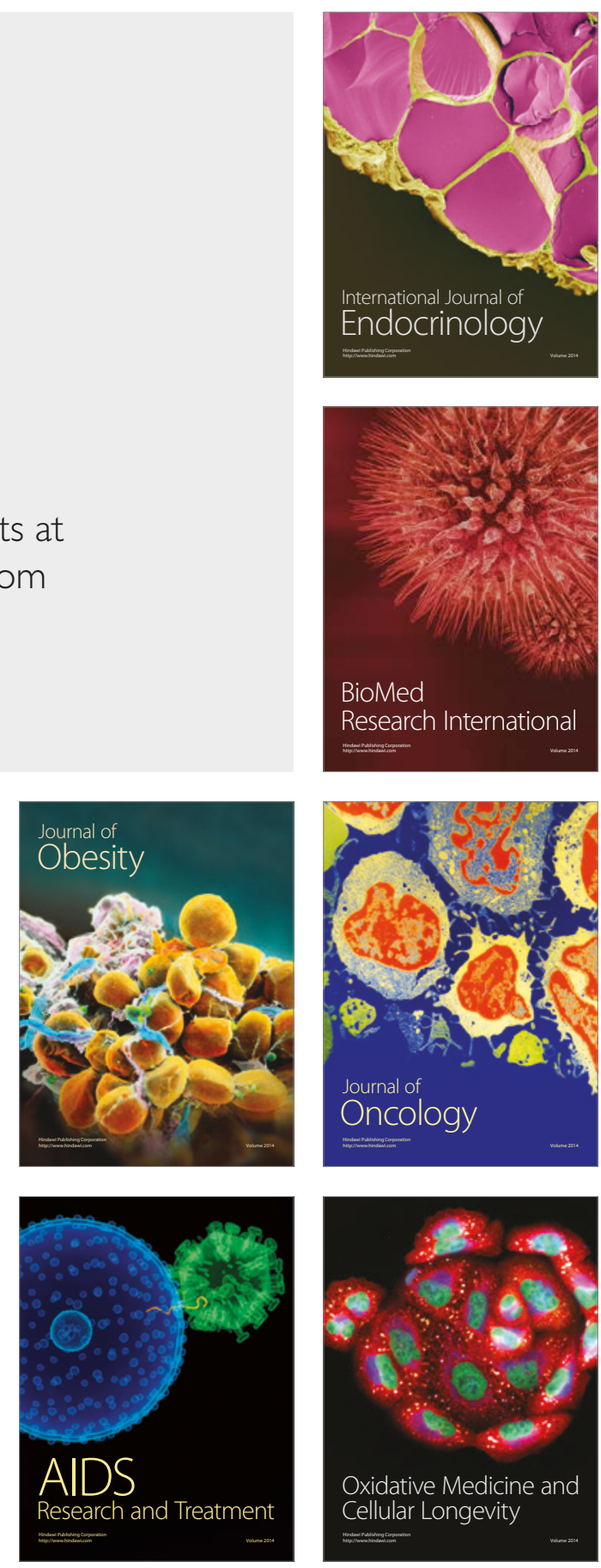